Case Report

\title{
A case of personal identification due to detection of rare DNA types from seminal stain
}

\author{
Hirofumi Tsutsumi ${ }^{1,2)}$, Toshinobu Komuro ${ }^{1,2)}$, Rei Mukoyama ${ }^{1)}$, Hikaru Izawa ${ }^{1,2)}$, \\ Jian $\mathrm{Tie}^{3)}$ and Seisaku Uchigasaki ${ }^{3)}$ \\ ${ }^{1)}$ Department of Legal Medicine, Nihon University School of Dentistry, Tokyo, Japan \\ ${ }^{2}$ Division of Social Dentistry, Dental Research Center, Nihon University School of Dentistry, Tokyo, Japan \\ ${ }^{3)}$ Division of Legal Medicine, Department of Social Medicine, Nihon University School of Medicine, \\ Tokyo, Japan
}

(Received 10 April and accepted 23 July 2009)

\begin{abstract}
Following a rape incident in an apartment in Japan, we were requested to perform a DNA analysis on a body fluid stain left on a bath towel to determine whether it could be attributed to the suspect. The acid phosphatase and prostatic-specific antigen tests confirmed it to be a seminal stain. Based on the DNA analysis by autosomal and Y-chromosome short tandem repeat (STR) systems, no inconsistency was found with the profile of the suspect with African ancestry. In this case, allele 21 of DYS390 at the Y-STR locus was examined, as it is reported to have a distinctly lower frequency in the Japanese population. Furthermore, the haplotype combinations of Y-STR at the DYS389I, DYS389II and DYS390 loci are powerful for personal identification, as these have not yet been found in the Japanese population. (J Oral Sci 51, 645-650, 2009)
\end{abstract}

Keywords: Y-chromosome STR; STR typing; repeat structure; seminal stain; personal identification.

\section{Introduction}

Crimes are often left unreported. This is particularly the case for sex crimes due to their violent nature. Thus, in a

Correspondence to Dr. Hirofumi Tsutsumi, Department of Legal Medicine, Nihon University School of Dentistry, 1-8-13 KandaSurugadai, Chiyoda-Ku, Tokyo 101-8310, Japan

Tel: +81-3-3219-8129

Fax: +81-3-3219-8343

E-mail: tsutsumi@dent.nihon-u.ac.jp sex crime, elucidating the actual events of the incident is difficult. However, an increasing number of sex crime victims are courageously reporting to the police, thereby improving arrests rates of criminals.

In this case of a rape occurring at the victim's apartment, we successfully identified the suspect through DNA analysis of a body fluid stain collected on a bath towel. In similar cases, such analyses have already been reported (1-6), but they rarely lead to successful identification. Our success is mainly due to the fact that the detected DNA types were all identical and that the analyzed gene region had a repeat structure that is rarely seen in the Japanese population $(7,8)$.

\section{Case Summary}

The victim was living alone in an apartment in Japan. The suspect supposedly hid in front of the apartment and waited for her to return home, at which time he pushed her inside and raped her. Immediately following the incident, the assailant forced the victim to take a shower. However, instead of washing off the seminal fluid, she wiped it off with a bath towel that she kept as evidence for the police.

As the assailant was described as being of African ancestry, a suspect was easily found and detained. A DNA analysis was performed on the seminal stain to determine whether it belonged to the suspect.

Sample

\section{Sample and Methods}

The body fluid from a stain on the bath towel obtained 
from the rape victim's home was used for seminal and DNA analyses. It should be noted that the sample from the stain also contained body fluid from the victim.

\section{Seminal stain inspection}

\section{Detection of acid phosphatase}

The fluid material of the stain was transferred to a large piece of filter paper wet with distilled water, by pressing the paper against the towel. An appropriate amount of SM Reagent (Wako Pure Chemical Industries, Ltd., Osaka, Japan), prepared by diluting 5 times with citrate buffer solution, was placed in a glass spray bottle, and sprayed on the filter paper (9).

2. Detection of prostatic-specific antigen

A PSA Semiquant Test (Seratec Ges. f. Biotechnologie $\mathrm{mbH}$, Göttingen, Germany) was carried out on the stain to detect prostatic-specific antigens in serum using a colloidal gold chromatographic immunoassay (10). First, a $5-\mathrm{mm}^{2}$ square was cut from the towel containing the stain and placed in a microtube. To extract the fluid material from the towel, $500 \mu \mathrm{l}$ of phosphate buffered saline ( $\mathrm{pH}$ 7.4) $\left(\right.$ Gibco $^{\circledR}$, Invitrogen, Corp., CA USA) was added. Next, $250 \mu \mathrm{l}$ of the extracted solution was dropped in a sample well, and the presence of prostatic-specific antigens was determined after $15 \mathrm{~min}$.

\section{DNA extraction from the seminal stain}

Another $5-\mathrm{mm}^{2}$ square containing the seminal stain was processed with Proteinase $\mathrm{K}$, and the DNA was extracted using QIAamp EZ1 DNA Tissue kit (Qiagen, Hilden, Germany). DNA was also extracted from blood samples of the victim and suspect using QIAamp DNA Mini kits (Qiagen).

\section{DNA typing of autosomal short tandem repeat (STR) loci}

Autosomal STR typing of the blood samples obtained from both the victim and suspect as well as from the seminal stain was performed using AmpFlSTR ${ }^{\circledR}$ Identifiler PCR Amplification kits (Applied Biosystems, Foster, CA, USA), for the following 15 loci: D8S1179, D21S11, D7S820, CSF1PO, D3S1358, TH01, D13S317, D16S539, D2S1338, D19S433, vWA, TPOX, D18S51, D5S818, and FGA. At the same time, the amelogenin locus was also examined to identify the sex. The ABI Prism ${ }^{\mathrm{TM}} 310$ Genetic Analyzer and GENESCAN Software (Applied Biosystems) were used for analysis.

\section{DNA typing of Y-chromosome STR loci}

Typing of Y-chromosome STR loci was performed using AmpFlSTR ${ }^{\circledR}$ Yfiler kits (Applied Biosystems), for the following 16 loci: DYS456, DYS389I, DYS390, DYS389II, DYS458, DYS19, DYS385a/b, DYS393, DYS391, DYS439, DYS635, DYS392, YGATAH4, DYS437, DYS438, and DYS448. ABI Prism ${ }^{\mathrm{TM}} 310$ Genetic Analyzer and GENESCAN Software (Applied Biosystems) were used for analysis.

\section{Analysis of repeat structures at DYS389II and DYS390 loci}

Following PCR amplification of both the suspect's blood sample and seminal stain, polyacrylamide gel electrophoresis (PAGE) was conducted for DYS389II and DYS390 loci. After purifying the obtained DNA bands and then sequencing them using the ABI Prism ${ }^{\text {TM }} 310$ Genetic Analyzer, their repeat structures were analyzed.

\section{Results}

\section{Seminal stain inspection}

1. Detection of acid phosphatase

On the filter paper containing the fluid material from the bath towel, several purple spots were visible following the application of the SM Reagent, indicating acid phosphatase was present. These spots were therefore areas on the towel most likely containing seminal fluid.

2. Detection of prostatic-specific antigens

A solution extracted from the area of the stain containing acid phosphatase was further examined using a PSA Semiquant test. The test presented a positive line (Fig. 1), indicating a specific antigen-antibody reaction. Therefore, the stain on the bath towel was confirmed to contain human sperm.

\section{DNA typing of autosomal STR loci}

A typing test for 15 loci of autosomal STR obtained from the seminal stain on the bath towel successfully revealed the DNA types of all corresponding loci. The electrophoretic profiles of D5S818 and FGA loci are shown as examples in Fig. 2. As indicated in Table 1, the DNA types of the 15 loci contained two to four alleles per locus. DNA typing was also performed on the 15 loci using the blood samples of the victim and suspect. One to two alleles were detected at each locus; all alleles found in the sample from the seminal stain were detected.

\section{DNA typing of Y-chromosome STR loci}

A typing test for 16 loci of Y-chromosome STR obtained from the seminal stain on the bath towel successfully revealed the DNA types of all corresponding loci. Figure 3 shows representative electrophoretic profiles of GATA, DYS437, DYS438, and DYS448 loci. Table 2 contains the alleles detected at the 16 loci. DNA typing of the suspect's 
blood sample was also examined, revealing identical alleles with that found in the seminal stain.

\section{Analysis of repeat structures at DYS389II and DYS390 loci}

Analysis of DYS389II and DYS390 loci confirmed the same repeat structure was found in both the suspect's blood sample and the seminal stain: (CTGT) ${ }_{6}(\mathrm{CTAT})_{13}$ $(\mathrm{CTGT})_{3}(\mathrm{CTAT})_{9}$ and $(\mathrm{CTGT})_{8}(\mathrm{CTAT})_{8}(\mathrm{CTGT})_{1}(\mathrm{CTAT})_{4}$, respectively $(7,8)$. Since the sum of the all the repeats was 31 for DYS389II locus and 21 for DYS390 locus, both samples were analyzed as Type 31 and Type21, respectively.

\section{Discussion}

DNA analysis is currently regarded as an indispensable tool for criminal investigations. In the case of sex crimes, DNA analysis can provide valuable evidence that is essential for personal identification of suspects $(11,12)$. That is, DNA typing of body fluid obtained from a crime scene and that from a suspect can be compared for confirmation of personal identification. Moreover, when a difference such as race has been identified, structure analysis of a specific gene region can provide further evidence of personal identification.

This rape case is considerably different from other cases $(1-3,13,14)$ That is, the victim collected the body fluid from the assailant on a bath towel for evidence.

Due to the victim's actions, the bath towel containing the seminal fluid could be analyzed for personal identification of the assailant. Based on the positive result of the SM Reagent test, the stain most likely consisted of semen. Subsequently, a serum PSA test detected the presence of prostatic-specific antigen, confirming that the stain actually contained human semen.

For DNA analysis of the seminal stain, typing of autosomal STR loci was performed. The stain was confirmed to contain DNA from two or more individuals, as two to four alleles were detected at each of the 15 loci examined and both the $\mathrm{X}$ and $\mathrm{Y}$ peaks were observed upon examining the amelogenin locus. These alleles were therefore assumed to be that of the victim and suspect. Comparison of the DNA types found in the blood samples of the victim and suspect confirmed the identity of the suspect (Fig. 2 and Table 1).

Based on the allele frequency data of 1,350 Japanese individuals (15), many of the allele types found in the suspect at the loci of D21S11, D7S820, CSFIPO, D13S317, D19S433, D18S51 and FGA were rare among the Japanese population (0.001-0.087), while those found in the victim were typical. By focusing on the allele frequency at each of the 15 loci examined in our analysis, the personal identification can be limited to individuals with exactly the same DNA types, and could potentially achieve 100\% accuracy of a single person from the world population.

While mitochondrial DNA is maternally inherited (16),

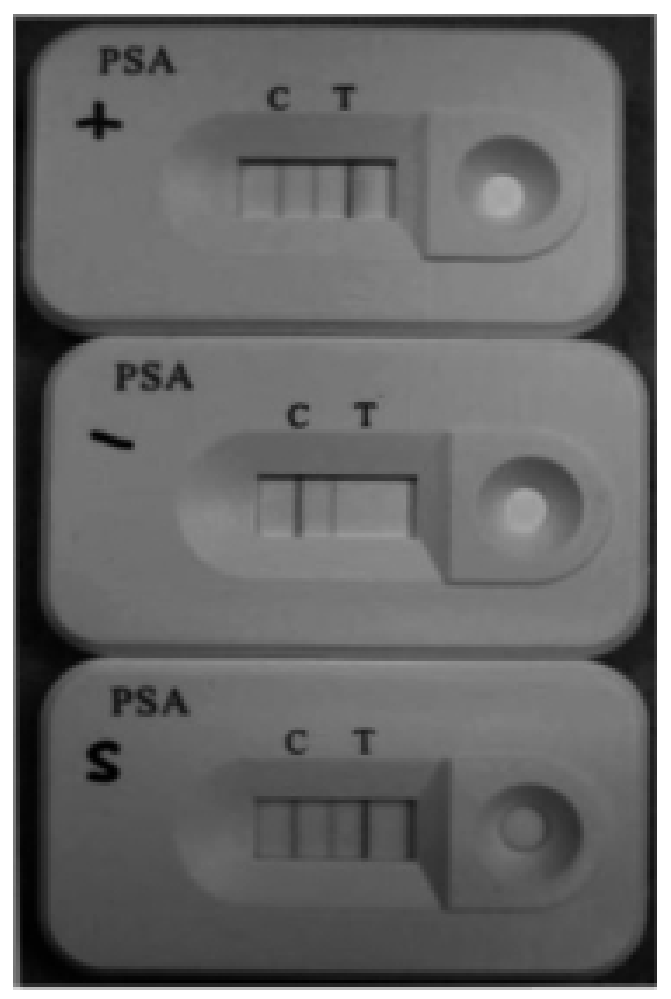

Fig. 1 Detection of prostatic-specific antigens using a PSA Semiquant Test.

+: positive sample, --: negative sample, S: stain sample; $\mathrm{C}$ : control line, $\mathrm{T}$ : test line.

A positive line was observed, indicating a specific antigen-antibody reaction.

Table 1 The autosomal STR typing using AmpFlSTRR Identifiler PCR Amplification Kits

\begin{tabular}{lccc}
\hline Locus & Seminal stain on a bath towel & Victim's blood & Suspect's blood \\
\hline D8S1179 & $13,14,17$ & $13-17$ & $14-14$ \\
D21S11 & $29,30,31,32.2$ & $29-30$ & $31-31.2$ \\
D7S820 & $8,11,13$ & $8-11$ & $13-13$ \\
CSF1PO & $9,11,12$ & $11-12$ & $9-11$ \\
D3S1358 & $15,16,17$ & 15 & $16-17$ \\
TH01 & $6,7,9$ & $6-7$ & $7-9$ \\
D13S317 & $9,11,13$ & $9-11$ & $11-13$ \\
D16S539 & 11,12 & $11-12$ & $11-12$ \\
D2S1338 & $17,23,27$ & $17-27$ & $23-23$ \\
D19S433 & $12.2,13,14$ & $13-14$ & $12.2-12.2$ \\
vWA & 16,17 & 17 & $16-17$ \\
TPOX & 8,9 & 9 & $8-9$ \\
D18S51 & $13,14.2,15,19$ & $13-15$ & $14.2-19$ \\
D5S818 & $11,12,13$ & 11 & $12-13$ \\
FGA & $20,22,24,28$ & $22-24$ & $20-28$ \\
Amelogenin & $X Y$ & $X$ & $X Y$ \\
\hline
\end{tabular}



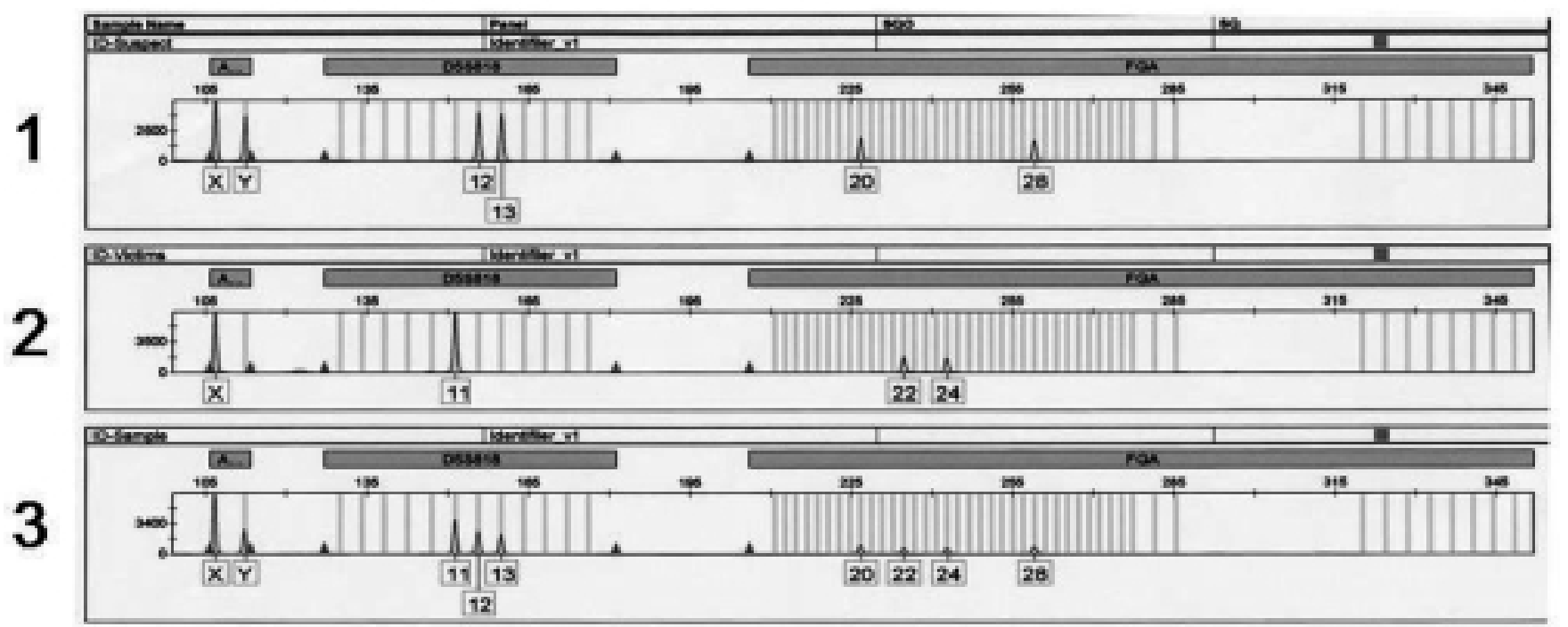

Fig. 2 DNA typing of autosomal short tandem repeat (STR) loci.

1: suspect, 2: victim, 3: SM-positive stain sample.

Autosomal STR typing of blood samples obtained from both the victim and suspect as well as the seminal stain was performed using AmpFISTR ${ }^{\circledR}$ Identifiler PCR Amplification kits (Applied Biosystems), for the following 15 loci: D8S1179, D21S11, D7S820, CSFIPO, D3S1358, TH01, D13S317, D16S539, D2S1338, D19S433, vWA, TPOX, D18S51, D5S818 and FGA. The electrophoretic profiles of D5S818 and FGA loci are shown.

Table 2 The typing of the Y-chromosome STR loci using AmpFlSTRR Yfiler Kits

\begin{tabular}{lcc}
\hline Locus & Seminal stain on a bath towel & Suspect's blood \\
\hline DYS456 & 15 & 15 \\
DYS3891 & 12 & 12 \\
DYS390 & 21 & 21 \\
DYS389II & 31 & 31 \\
DYS458 & 17 & 17 \\
DYS19 & 15 & 15 \\
DYS385a/b & $17 / 17$ & $17 / 17$ \\
DYS393 & 13 & 13 \\
DYS391 & 10 & 10 \\
DYS439 & 13 & 13 \\
DYS635 & 22 & 22 \\
DYS392 & 11 & 11 \\
GATA & 12 & 12 \\
DYS437 & 14 & 14 \\
DYS438 & 11 & 11 \\
DYS448 & 21 & 21 \\
\hline
\end{tabular}

Y-chromosome STR loci are paternally inherited (4). Parentage determination relies on these genetic rules, as evident in this case, where the genetic material was obtained from a stain mixed with seminal and vaginal fluids. In this case, analysis of Y-chromosome STR loci can provide highly reliable evidence for identifying suspects $(1-3,13,14)$. Moreover, this analysis detects only one allele at each locus of the Y-chromosome STR, thereby allowing for identification of even multiple assailants (3).
Typing of Y-chromosome STR loci therefore has great potential. In this case, a typing test of 16 Y-chromosome STR loci detected from the seminal stain successfully revealed the DNA types of all corresponding loci. Comparison of the DNA types found in the suspect's blood sample with that in the seminal stain found that all alleles were identical (Fig. 3 and Table 2). Based on the allele frequency data of 937 Japanese individuals (17), the alleles in the suspect's DNA at the loci of DYS390, DYS385a and DYS448 are rarely found in the Japanese population (0.003-0.59). Even after crosschecking the data of Hashiyada et al. (17) with the DNA types at the 16 loci, we found none of the 932 Japanese individuals shared the same haplotype with the suspect.

We also conducted an analysis on the repeat structures at DYS389II and DYS390 loci of the Y-chromosome STR, based on the reports by Komuro et al. $(7,8)$, which indicate that these repeat structures have length polymorphisms and that a detailed examination of the sequence of each allele can be useful for personal identification.

The repeat structure of DYS389II locus is composed of a complex of repeated CTGT and CTAT sequences, as in $(\mathrm{CTGT})_{\mathrm{p}}(\mathrm{CTAT})_{\mathrm{q}}(\mathrm{CTGT})_{3}(\mathrm{CTAT})_{\mathrm{r}}$. According to Komuro et al. $(7,8)$, the number of repeats found in the Japanese population are as follows: $\mathrm{p}=4$ or $5, \mathrm{q}=10$ to 14 , and $\mathrm{r}$ $=8$ to 12 . The repeat structures observed in the seminal stain and the suspect's blood sample were equivalent: 
$(\mathrm{CTGT})_{6}(\mathrm{CTAT})_{13}(\mathrm{CTGT})_{3}(\mathrm{CTAT})_{9}$; the sum of the repeats was 31, and DYS389II locus from the two samples was classified as Type 31 . Two types coexist at DYS389 locus, DYS389I and II, corresponding to Type 12 and Type 31, respectively, which was found for the Y-chromosome STR loci in this case. While Komuro et al. (8) does not mention the existence of Type 31, Hashiyada et al. (17) states that the allele frequency of Type 12 and Type 31 among the Japanese population was 0.172 and 0.192 , respectively. The public Y-Filer Haplotype Database (http://www. appliedbiosystems.com/yfiler database/; provided by Applied Biosystems) contains information gathered from 330 individuals of Asian origin and gives an allele frequency is 0.4758 for Type 12 and 0.0697 for Type 31, which seem to be relatively high. None of the 330 individuals in the Y-Filer Haplotype Database were identified as having Type 12 and Type 31 as haplotypes, which was the same for the results based on Hashiyada et al. (17).

On the other hand, the repeat structure of DYS390 locus was $(\mathrm{CTGT})_{8}(\mathrm{CTAT})_{8}(\mathrm{CTGT})_{1}(\mathrm{CTAT})_{4}$ and therefore
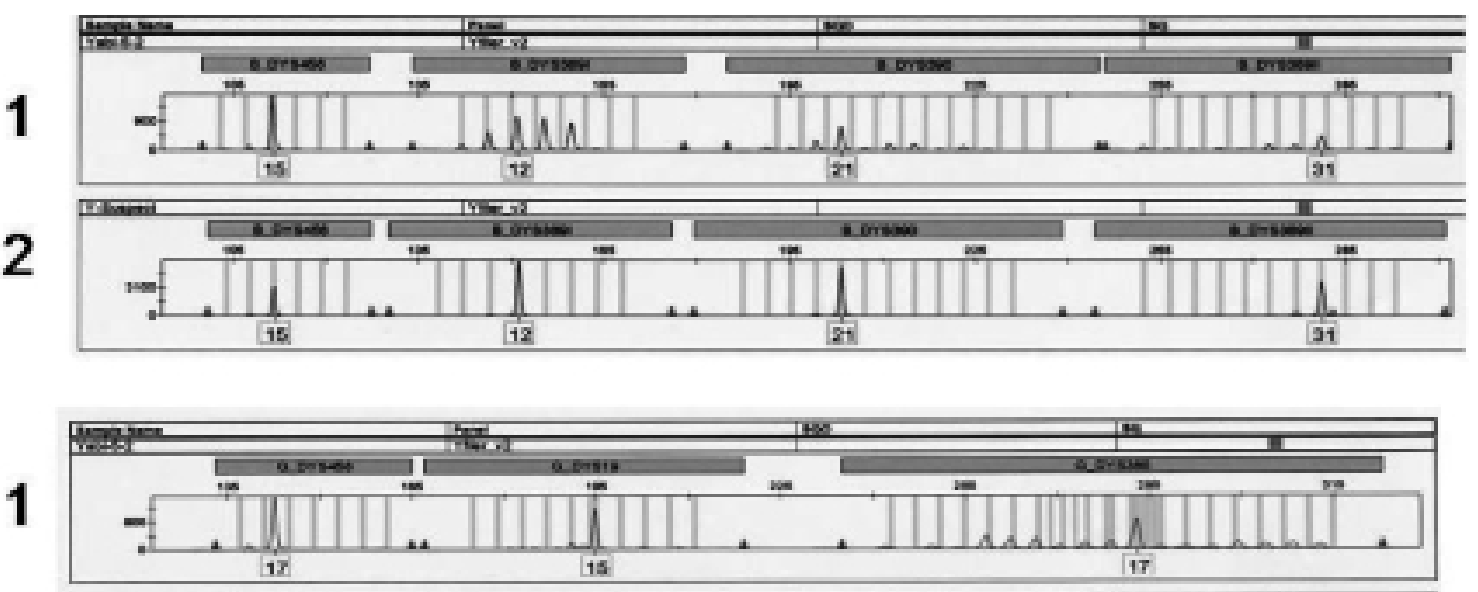

2
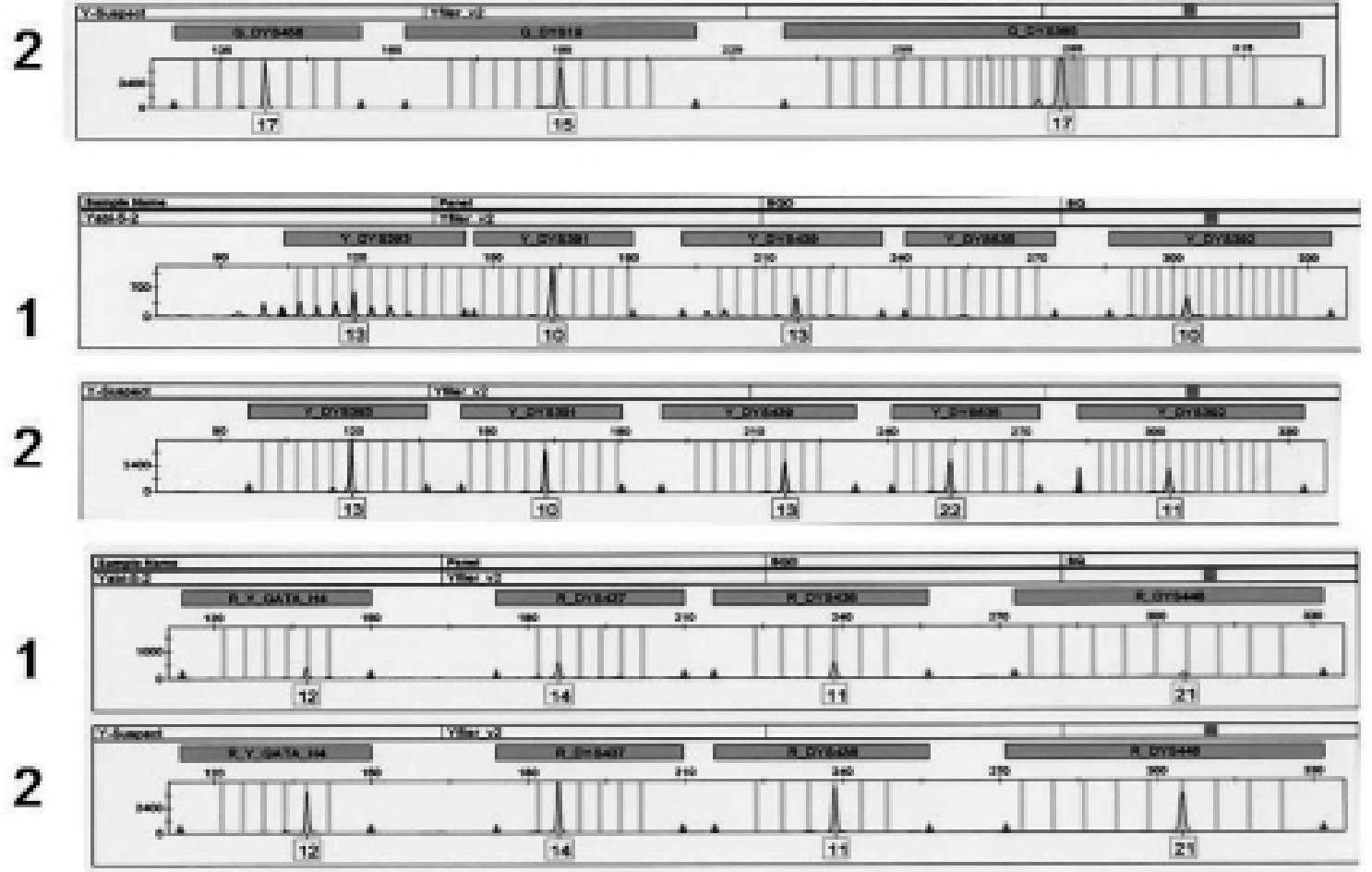

Fig. 3 DNA typing of Y-chromosome STR loci.

1: SM-positive stain sample, 2: suspect.

Typing of Y-chromosome STR loci was performed using AmpFISTR ${ }^{\circledR}$ Yfiler kits (Applied Biosystems), for the following 16 loci: DYS456, DYS389I, DYS390, DYS389II, DYS458, DYS19, DYS385a/b, DYS393, DYS391, DYS439, DYS635, DYS392, YGATAH4, DYS437, DYS438 and DYS448. 
classified as Type 21, based on the sum of the repeats. The allele frequency was 0.003 based on Hashiyada et al. (17) and 0.0121 based on the Y-Filer Haplotype Database, suggesting that Type 21 at DYS390 locus is extremely rare for the Japanese population.

With respect to the allele frequency of the haplotypes that were shown at DYS389I, DYS389II and DYS390 loci in DNA obtained from the seminal stain, data based on Hashiyada et al. (17) found 0 individuals with the same frequency, whereas three out of 3,561 $(0.084 \%)$ were found based on the Y-Filer Haplotype Database; these three subjects were of African ancestry. No individual of Asian origin has been reported to have these haplotypes at the corresponding loci, suggesting that this DNA type is very rare in Japan. In fact, in this case, the police later identified the suspect as an African with Nigerian nationality.

\section{Acknowledgments}

This work was supported in part by a Grant from the Dental Research Center, Nihon University School of Dentistry in 2007, by the Sato Fund, Nihon University School of Dentistry in 2007 and 2009.

\section{References}

1. Gross AM, Liberty AA, Ulland MM, Kuriger JK (2008) Internal validation of the AmpFISTR Yfiler amplification kit for use in forensic casework. J Forensic Sci 53, 125-134.

2. Hall A, Ballantyne J (2003) The development of an 18-locus Y-STR system for forensic casework. Anal Bioanal Chem 376, 1234-1246.

3. Johnson CL, Giles RC, Warren JH, Floyd JI, Staub RW (2005) Analysis of non-suspect samples lacking visually identifiable sperm using a Y-STR 10-plex. J Forensic Sci 50, 1116-1118.

4. Mayntz-Press KA, Ballantyne J (2007) Performance characteristics of commercial Y-STR multiplex systems. J Forensic Sci 52, 1025-1034.

5. Torres Y, Flores I, Prieto V, López-Soto M, Farfán MJ, Carracedo A, Sanz P (2003) DNA mixtures in forensic casework: a 4-year retrospective study. Forensic Sci Int 134, 180-186.

6. Calacal GC, Delfin FC, Tan MM, Roewer L, Magtanong DL, Lara MC, Fortun R, De Ungria MC (2005) Identification of exhumed remains of fire tragedy victims using conventional methods and
autosomal/Y-chromosomal short tandem repeat DNA profiling. Am J Forensic Med Pathol 26, 285291.

7. Komuro T, Tsutsumi T, Mukoyama R (2001) Ychromosomal STR haplotypes by the repeat structure polymorphisms in Japanese population. Nippon Hanzaigaku Zasshi 67, 197-201.

8. Komuro T, Tsutsumi T, Mukoyama R, Nakamura M (1998) Repeat structure of DYS389 locus. Nippon Houigaku Zasshi 52, 227-232. (in Japanese)

9. Wako Pure Chemical Industries, Ltd (2005) SM reagent. Osaka. (in Japanese)

10. SERATEC DIAGNOSTICA ${ }^{\circledR}$ Ges. F Biotechnologie mbH (2005) SERATEC ${ }^{\circledR}$ PSA SEMIQUANT. Göttingen.

11. Hall A, Ballantyne J (2003) Novel Y-STR typing strategies reveal the genetic profile of the semen donor in extended interval post-coital cervicovaginal samples. Forensic Sci Int 136, 58-72.

12. Fukshansky N, Bär W (2005) DNA mixtures: biostatistics for mixed stains with haplotypic genetic markers. Int J Legal Med 119, 285-290.

13. Ayadi I, Mahfoudh-Lahiani N, Makni H, AmmarKeskes L, Rebaï A (2007) Combining autosomal and Y-Chromosomal short tandem repeat data in paternity testing with male child: methods and application. J Forensic Sci 52, 1068-1072.

14. Irwin JA, Edson SM, Loreille O, Just RS, Barritt SM, Lee DA, Holland TD, Parsons TJ, Leney MD (2007) DNA identification of "Earthquake McGoon" 50 Years postmortem. J Forensic Sci 52, 1115-1118.

15. Yoshida K, Mizuno N, Fujii K, Senju H, Sekiguchi K, Kasai K, Sato H (2003) Japanese population database for nine STR loci of the AmpFISTR Profiler kit. Forensic Sci Int 132, 166-167.

16. Anderson S, Bankier AT, Barrell BG, de Bruijn MHL, Coulson AR, Drouin J, Eperon IC, Nierlich DP, Roe BA, Sanger F, Schreier PH, Smith AJH, Staden R, Young IG (1981) Sequence and organization of the human mitochondrial genome. Nature 290, 457-465.

17. Hashiyada M, Umetsu K, Yuasa I, Tamura A, Miyoshi A, Suzuki K, Kashimura S, Funayama M (2006) 17 Y-chromosomal STR haplotypes in Japanese. In: DNA polymorphism Vol.14, Japanese Society for DNA Polymorphism Research, Kishi K eds, Toyoshoten, Tokyo, 113-115. (in Japanese) 